\title{
Study of Active Pharmaceutical Ingredient (API) Formulations with Nonlinear Optical (NLO) Microscopy
}

\author{
Azhad U. Chowdhury ${ }^{1}$, Scott J. Toth ${ }^{1}$, Lynne S. Taylor2 and Garth J. Simpson ${ }^{1}$ \\ 1. Purdue University, Department of Chemistry, West Lafayette, IN-47907, USA \\ 1. Department of Industrial and Physical Pharmacy, Purdue University, West Lafayette, IN- 47907, USA
}

Whether an active pharmaceutical ingredient is present as an amorphous or crystalline solid can have a significant impact on its bioavailability, solubility, dissolution rate, physical stability and necessary storage conditions (i.e. temperature and humidity)[1,2]. These physical properties can also be affected by formulation and processing of the APIs in excipient matrices. Therefore, physical studies of a pure API solid, as well as its formulation, are needed.

Many analytical techniques are used to study the solid-state form of an API. Traditional analytical techniques for solid-state analysis include differential scanning calorimetry (DSC), thermogravimetric analysis (TGA), Fourier transform infrared spectroscopy (FT-IR), Raman spectroscopy, and powder Xray diffraction (PXRD)[3]. Each of these techniques has its own advantages and drawbacks associated with it for solid-state analysis. However, in the case of formulations when the crystal loading of APIs is low, all of these techniques suffer from the low resolution and relatively high detection limits due to interferences from the excipient matrix. Currently, the X-ray diffraction is able to detect low levels of crystallinity $(\sim 0.2 \%)[4]$, but it requires X-rays fluxes generated by synchrotron sources to achieve this level of sensitivity, which is not suitable for routine in-house analysis. Furthermore, the aforementioned methods only provide ensemble-averaged properties, with no microscopic information (e.g., crystal size distributions, crystal nucleation kinetics, etc.), which can impact mechanistic studies and the development of predictive models.

Second harmonic generation (SHG) microscopy has been recently developed as a tool for selective detection of low crystal loading, detection of crystal formation at early stages and for the study the nucleation and crystal growth kinetics. SHG is a second order nonlinear optical process in which the frequency of the incident light is doubled. SHG is symmetry forbidden in centrosymmetric media, and consequently amorphous solids, liquids, solvated molecules. By this symmetry argument only the chiral crystals can mitigate the criteria to be selectively detected under the SHG microscope[5]. Two-photon excited ultraviolet fluorescence (TPE-UVF) is a complimentary method to SHG, generating contrast from intrinsic fluorescence from aromatic molecules, irrespective of crystallinity[6,7]. As the majority of APIs are aromatic, TPE-UVF provides a selective detection method for APIs. TPE-UVF and UVSHG (excitation with $530 \mathrm{~nm}$ rather than $800 \mathrm{~nm}$ ) microscopy can be performed simultaneously to visualize the size and shape distribution of API crystals in excipient matrices. UV-SHG is used for two reasons 1) it can be performed simultaneously with TPE-UVF and 2) A resonance enhancement is observed in the SHG signal from the excitation of aromatic groups. Kinetic studies can also be performed using this combined approach to study the formation and degradation of API crystals under different conditions. 
The capability of SHG microscopy to provide high image contrast of API crystals distributed throughout turbid excipient matrices is explored. Naproxen was homogeneously dispersed in PEG above the melting temperature in a 1:20 ratio, then flash-cooled. Upon temperature elevation, naproxen crystallization was monitored by SHG microscopy, with volume maps acquired for 9 planes through the sample, with $30 \mathrm{~s}$ exposures per plane. Both nucleation kinetics and overall crystallization kinetics are obtained independently. The crystal morphology and spatial distribution were found to be highly dependent on loading and temperature. In contrast, conventional bench-top approaches for characterizing crystallinity can routinely exhibit detection limits for crystallinity around the $5 \%$ value asymptotically approached [8].

SHG microscopy and UV-SHG coupled with TPE-UVF were explored for selective detection of model APIs in mixtures of common excipients. Crystalline lactose is found to generate the most interference for SHG measurements, representing $\sim 12 \%$ of the SHG activity of an API (griseofulvin) for conventional SHG and $\sim 2 \%$ for UV-SHG [9]. The enhanced selectivity is attributed to resonanceenhanced SHG from the aromatic moiety in griseofulvin. Investigations on cleaved Cialis tablets allowed measurements of tadalafil distributions to depths of $140 \mu \mathrm{m}$ while preserving high $\mathrm{S} / \mathrm{N}$ and image quality. The ability to selectively detect and image APIs within excipient matrices should help lay the foundation for applications involving API distribution and homogeneity studies within tablets and formulations.

References:

[1] Hancock, B. C. \& Zograf, G., Characteristics and significance of the amorphous state in pharmaceutical systems. Journal of Pharmaceutical Sciences 86 (1997), p.1-12.

[2] Andronis, V. \& Zografi, G., The molecular mobility of supercooled amorphous indomethacin as a function of temperature and relative humidity. Pharm. Res. 15 (1998), p. 835-842.

[3] Robert T. B., et al., Solid-state NMR spectroscopy in pharmaceutical research and analysis. Trends in Analytical Chemistry 25(2006), p.977-984.

[4] Moukhametzianov, R., et al., Acta Crystallogr., Sect. D: Biol. Crystallogr., 64 (2008), p. 158-166.

[5] Kissick, D.J., et al., Analytical Chemistry, 82 (2010), p. 491-497.

[6] Madden, J.T., et al., Acta Crystallogr., Sect. D: Biol. Crystallogr., 67 (2011), p. 839-846.

[7] Haupert, L.M., et al., Acta Crystallographica Section D, 68 (2012), p. 1513-1521.

[8] Qing Zhu., et al., J. Phys. Chem, 117 (2013), p. 1494-1500.

[9] Toth S. J., et al., Selective Imaging of Active Pharmaceutical Ingredients in Powdered Blends with Common Excipients Utilizing Two-Photon Excited Ultraviolet-Fluorescence and Ultraviolet-Second Order Nonlinear Optical Imaging of Chiral Crystals, Analytical Chemistry, 84 (2012), p. 5869-5875 